\title{
Routing Protocols for VANETs: A Taxonomy, Evaluation and Analysis
}

Amina Bengag*, Asmae Bengag, Mohamed Elboukhari

MATSI lab, ESTO, Mohammed First University, Oujda, Morocco

\begin{tabular}{l} 
A R T I C L E I N F O \\
\hline Article history: \\
Received: 21 September, 2019 \\
Accepted: 27 December, 2019 \\
Online: 15 January, 2020 \\
\hline Keywords: \\
VANETs \\
Routing protocols \\
SUMO \\
NS-3 \\
OSM \\
Urban scenario \\
Greedy forwarding \\
Angle direction
\end{tabular}

\begin{abstract}
A B S T R A C T
VANET as a subclass of MANET is composed of a set of vehicles equipped with wireless transceivers, to build dynamic networks without the need of any pre-existing infrastructure. Over the last few decades, the area of routing protocols in VANETs has been extensively studied. Nevertheless, this area remains even more challenging due to some features of VANETs, such as the high speed of vehicles, the often-disconnected links and the particular mobility pattern. Routing protocols in VANETs could be splitted into four categories: topology, position, multicast and broadcast-based routing protocols. In this paper, we provide a novel detailed taxonomy of routing protocols in VANETs then we present the advantages and drawbacks of each category. Moreover, we clear up the techniques adopted by each of the most popular routing protocols based on the vehicles' position and the topology of the networks. To explore the strengths and weaknesses of each routing protocols, basing on their suitability for VANET, we implemented them by using SUMO and NS3 as simulation tools applied on a real street map of Oujda city. We have extracted the used map from Open Street Map (OSM). Finally, we present our future works used for optimizing the greedy forwarding technique that is adopted by some position-based routing protocols in VANETs. Our suggested technique is based on the angle direction and three other important parameters of the relaying node.
\end{abstract}

\section{Introduction}

In this paper, we review and provide an extension version of previous work originally presented in the 5th International IEEE Congress on Information Sciences and Technology (IEEECiST'18) [1], in addition to some new enhancements. It is about routing protocols in Vehicular Ad-hoc Networks.

Vehicular networks have become the heart of the Intelligent Transport Systems or ITS in short, to improve the road traffic safety and to make a comfortable driving environment for drivers and passengers. In order to establish a communication in VANET, there is no need to any pre-existing infrastructure; this kind of networks consist of a set of vehicles containing wireless transceivers, named On-Board Unit (OBU). These OBUs help vehicles to exchange information between each other.

Compared to other sub-category of Mobile Ad-Hoc Networks, vehicular networks have many exclusive and specific features, such as diverse communication environments. Actually, in the rural environment, the issue of sparse connectivity is high and the traffic condition is simple; while for the city environment, the

*Corresponding Author: Amina BENGAG, bengag.amina@gmail.com density and the number of obstacles (such as building trees and others) are high, which make the traffic condition very complex. Besides, the high speed of vehicles could be the main cause of the high dynamic topology and the regularly link breakage issue as the second and third features of VANETS. The last one is the variable network density, which is caused by the fact that the traffic density varies. This later depends on the type of environments and the moment of the day, which is very high in rush hour and medium or low in the other time.

Due to the above-mentioned features of vehicular networks, the proposition of an effective routing technique remains even more challenging to overcome. In this paper, we give our proposed taxonomy of routing techniques in vehicular networks [2] into routing based on network's topology, vehicle's position, multicast technique and broadcast technique as is shown in Figure 1.

The main objective behind the presentation of this taxonomy is to specify the advantages and disadvantages of each routing protocols' class, then declare the most convenient categories to VANETs. Furthermore, this declaration will be proved by a comparison study that is provided under two simulation tools. We also introduce our future work in which we suggest a new strategy 


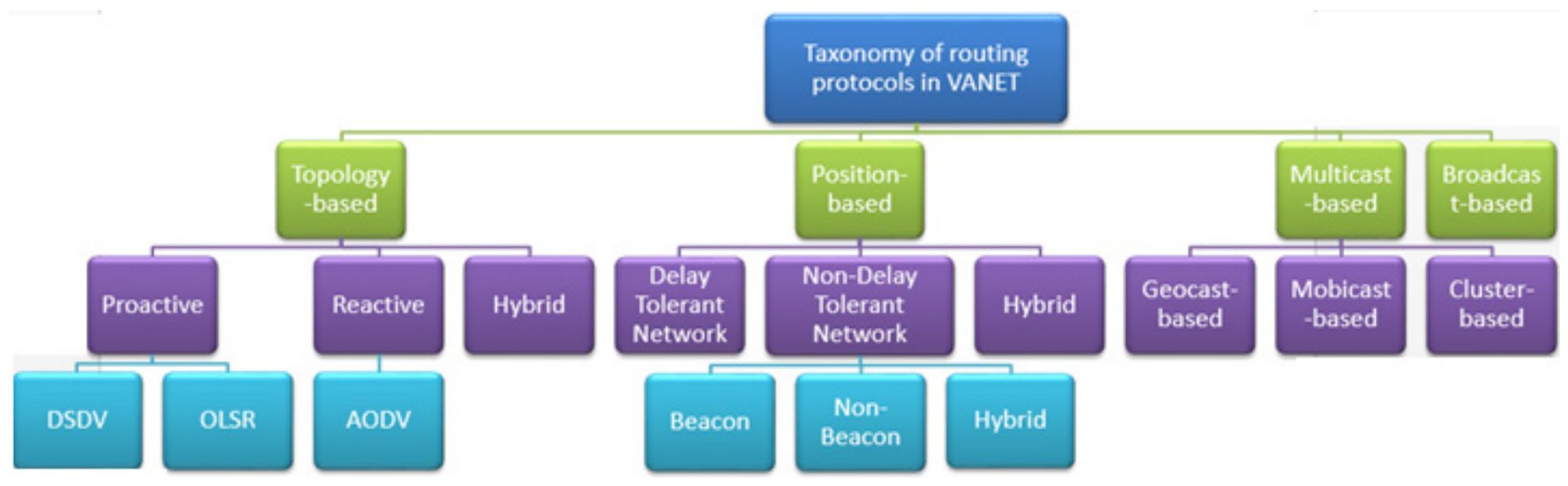

Figure 1 Taxonomy of VANETS routing protocols

to enhance the high-performed routing protocols, which belong to the category based on vehicles' position. This new strategy proved through theoretical analysis, is based on four parameters like velocity, the direction of vehicles, the density and the vehicles' position.

The rest of this paper is organized as follows: in section II, we outline the related works. In section III, we provide a detailed categorization of VANET's routing protocols and discuss their benefits and issues [2]. Section IV discusses in detail a comparison study of some routing techniques, based on the network topology and others on the physical position of vehicles by utilizing two simulation tools like NS3 as a network simulator and SUMO [1] as a road traffic simulator. Then, in section $\mathrm{V}$, we introduce our current work and the proposed technique to optimize the Greedy forwarding strategy in GPSR protocol. Finally, in section VI, we conclude the paper and we present some our future works.

\section{Previous work}

To select the best-suited protocol to Vehicular Networks, many studies have been conducted to evaluate and examine the performance of certain routing mechanism based on simulation tools. In Table 1, we present some of the previous works related to the mentioned above study. It could be concluded from the table that all works is based on ns- 2 or ns-3 as networks simulators, while some of them use also a specific road traffic simulators such as SUMO. However, in this work we provide a new study distinguish from those cited in Table I in many sides.

Basically, our work in this paper, focused on modeling the real details of VANETs network movement to examine the performance of certain routing protocols: DSDV, AODV, GPSR, OLSR, and GPCR. We extracted a part of a real road map from open street map (OSM) of a large street in Oujda city (Hay alQuds), instead of considering a grid map or a random map. This map was made to be as an input file in SUMO in order to create mobility and traffic files; those files will be used as an entry in NS3 for further analysis of network performance.

\section{Taxonomy of Routing Protocols in VANETs}

The regular link breakage issue of vehicular networks caused by the speed of vehicles that lead to the high dynamic topology in these networks; the mentioned problems make the task of transmitting data packet in vehicular networks a very challenging www.astesj.com one. Mainly, routing protocols in VANET can be classified into four categories [2] for $\mathrm{V} 2 \mathrm{~V}$ communication: based on the network's topology, based on the vehicles' position, based on multicast technique and finally routing based on broadcast technique.

Table 1 Related Works

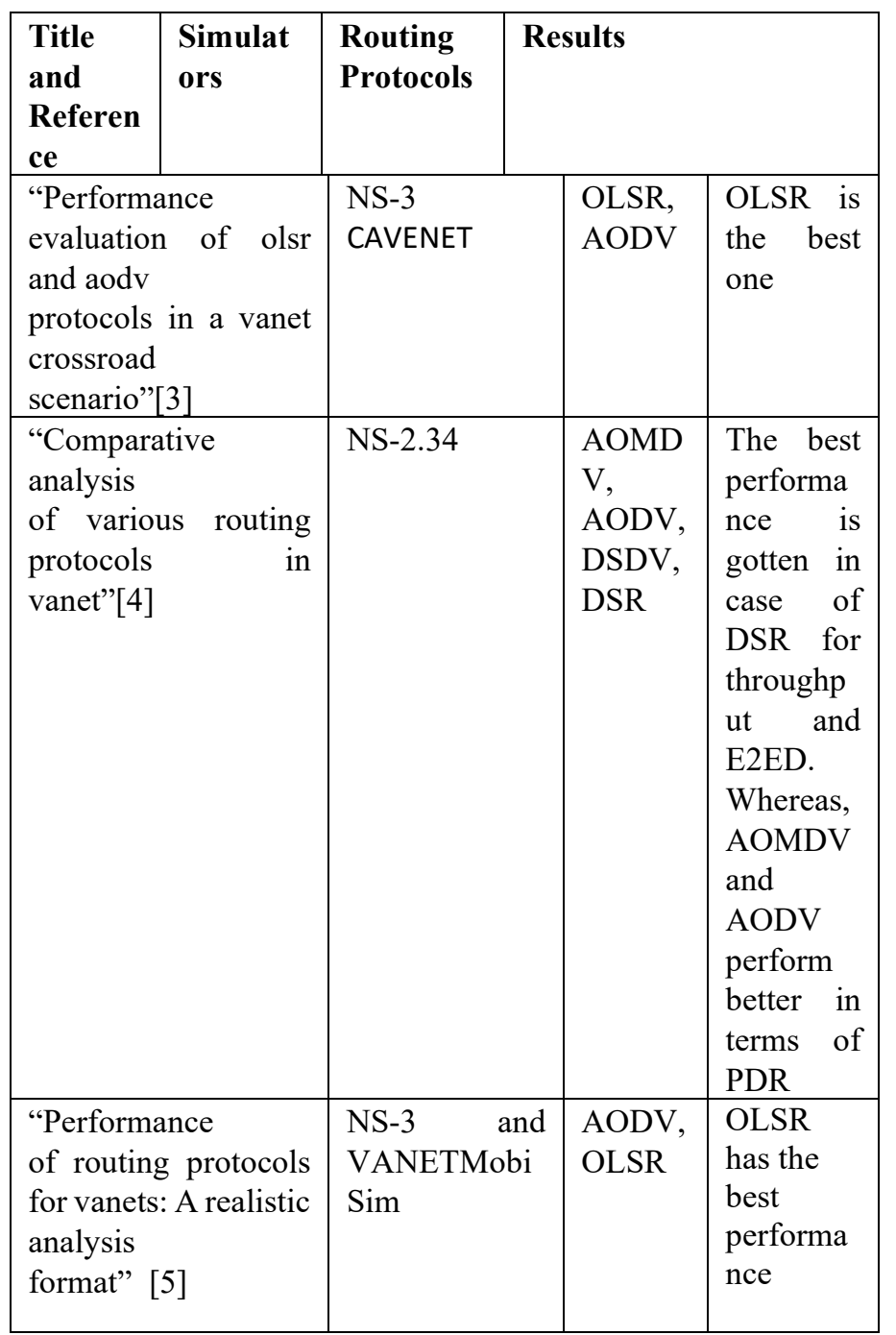




\begin{tabular}{|c|c|c|c|}
\hline $\begin{array}{ll}\text { "Scenario based } & \text { performance } \\
\text { analysis of aodv and } \\
\text { gpsr routing } \\
\text { protocols in r a } \\
\text { vanet"[6] }\end{array}$ & $\begin{array}{l}\text { NS-2.35 and } \\
\text { VANETMobi } \\
\text { Sim }\end{array}$ & $\begin{array}{l}\text { AODV, } \\
\text { GPSR }\end{array}$ & $\begin{array}{l}\text { AODV } \\
\text { outperfor } \\
\text { ms GPSR } \\
\text { in case of } \\
\text { PDR } \\
\text { metric } \\
\text { and } \\
\text { GPSR } \\
\text { behaves } \\
\text { better for } \\
\text { E2ED }\end{array}$ \\
\hline $\begin{array}{l}\text { "Performance } \\
\text { comparison of } \\
\text { position based } \\
\text { routing protocols for } \\
\text { vanets"[7] }\end{array}$ & $\begin{array}{l}\text { NS-2 and } \\
\text { VANETMobi } \\
\text { Sim }\end{array}$ & $\begin{array}{l}\text { GPSR, } \\
\text { GPCR }\end{array}$ & $\begin{array}{l}\text { GPCR is } \\
\text { the best } \\
\text { one }\end{array}$ \\
\hline $\begin{array}{l}\text { "Performance } \\
\text { Evaluation of OLSR } \\
\text { and } \\
\text { AODV in VANET } \\
\text { Cloud Computing } \\
\text { Using Fading Model } \\
\text { with SUMO } \\
\text { and NS3"[8] }\end{array}$ & $\begin{array}{l}\text { NS-3 and } \\
\text { SUMO }\end{array}$ & $\begin{array}{l}\text { AODV, } \\
\text { OLSR }\end{array}$ & $\begin{array}{l}\text { Almost } \\
\text { the same } \\
\text { performa } \\
\text { nce for } \\
\text { both } \\
\text { protocols }\end{array}$ \\
\hline
\end{tabular}

\subsection{Routing based on the network topology}

In this category, picking an itinerary between the source and the target node is based the existing link information in the network. The collected information could be done before any need of routing by the vehicle by utilizing proactive technique that is known by the table-driven technique. Moreover, for reactive technique, the information is searched when we need to transmit data; this procedure is also known by on-demand technique, in which the step of finding or maintaining an itinerary from source vehicle to destination is essential before any transmission.

In proactive technique, nodes collect and maintain updated routing data beforehand by periodically distributing routing tables throughout the network to all the nodes in its transmission range. The control messages in proactive routing are broadcasted continuously even if there is no data flow to send. Hence, the routing overhead is very high and the PDR is low consequently. The benefit of this category of routing protocols is that nodes can found routing information easily. DSDV, OLSR are two examples of proactive routing protocols.

In reactive technique, nodes start searching for a route only if they need to transmit data. Hence, they flood the network with Route Request packets (RREQ). AODV and DSR are two examples from this category. There is also one more type called hybrid protocol that associates reactive and proactive techniques, which make them more efficient. Zone Routing protocol (ZRP) is an example of such routing technique [9].

\subsection{Routing based on vehicle's Position [10]}

This class of routing protocols is also known as geographic protocols. They require the availability of a positioning system that provide the physical location of the participating vehicles in the relay selection process. The utility of this physical position information is identifying and making decision to select the next hop to route packet to the last destination. This technique reduces the routing overhead issue because it does not require any routing tables or exchange any information between them to establish routes. Here, it is not essential to identify the whole route to deliver the data packets that reduce the end-to-end delay. Based on several previous works, we can divide the routing based on vehicle's position into three sub-categories: routing adapted to Delay Tolerant Network (DTN), routing adapted to Non-Delay Tolerant Network (Non-DTN) and hybrid position-based routing.

Routing protocols for DTN: this category of routing techniques is considered as an efficient one for networks that have some specific features, such as high link breakage, limited bandwidth, power constraint and large scale. Those problems could be reduced by using a carry-and-forward technique, in which the transmitted vehicle stores the packet until it finds another vehicle moves into its vicinity. Vehicle-Assisted Data Delivery (VADD) [11] is an example of such routing technique.

Routing for Non-DTN: those protocols are characterized by the regularly link breakage issue that cause the lack of connectivity and a lack of instant end-to-end routes. The greedy approach is the process adopted by this routing to look for the next relaying vehicle. Actually, by utilizing this strategy, the packet is transmitted to the nearest neighbor of the destination. While the transmission failure could happen in anytime especially when a node finds no neighbors nearer to the destination than itself. In non-DTN protocols, we can find three sub-class; routing based on beacon that use a periodic hello message, others are beaconless, and hybrid protocols. GPSR and GPCR are an example of such routing technique considered as beacon-based protocols.

Hybrid position-based Routing protocols: this category of routing techniques could be considered as a combination of the two previous mentioned techniques. The main advantages of this category is reducing the control overhead and resolving the frequently link breakage problem in VANET. GeoDTN+Nav [12] is an example of such routing technique.

\subsection{Routing based on multicast technique [13]}

In routing based on multicast technique, the data packet is forwarded from a single source to a specific group of vehicles by using multi-hop communication technique. This protocol could be divided into three categories: protocols based on the geocast technique, based on Mobicast technique and those based on clusters.

- Routing based on Clusters [14] here, the vehicles share the same features such as the direction of mobility with almost the same velocity can form a cluster, and in each one a node will be elected to manage the communication into each cluster and between those clusters. This node is called a cluster head $(\mathrm{CH})$. Cluster based Routing (CBR) [15] is an example of this category. 


\section{A. Bengag et al. / Advances in Science, Technology and Engineering Systems Journal Vol. 5, No. 1, 77-85 (2020)}

- Geocast-based routing protocols [16] here, data is forwarded from a single vehicle as source to vehicles belonging to multicast group or zone of relevance (ZOR) based on the physical position of vehicles. One of the most known routing in this category is Inter-Vehicule Geocast (IVG) [17].

- Routing based on Mobicast technique [18] it is a kind of geocast-based protocol that are based in time as another factor in addition to the space. Actually, by using this method, data packets will be transmitted to all vehicles situated in certain geographic area at a specific time $t$ so called ZORt. One of the most popular routing in this class is Mobicast routing protocol with carry and forward [18].

\subsection{Routing based on broadcast technique [19]}

In the broadcast technique, the data packets will be transmitted to reach the whole vehicles in the network. This technique is usually used in vehicular networks to share some important information between vehicles, such as road conditions, traffic, weather and emergency event. Based on several previous works [20], [21], we can divided this class of routing protocols into three categories as shown in Figure 2. These categories could have several common properties such as information type, information source, broadcast decision and orthogonal enhancements.

The three classes of routing protocols based on broadcast techniques are:

- The deterministic that is based on the topology of the network, the position and the velocity of the vehicles to rebroadcast the data packets.

- The probabilistic in which a vehicle will rebroadcast a data packet with a certain probability fixed in priory or adapted periodically.

- The hybrid broadcast that is a combination of deterministic and probabilistic techniques. In fact, the hybrid broadcast technique uses the deterministic technique to compute the most convenient broadcast probabilities.

Distribution Vehicular broadCAST (DV-CAST) [22], Distribution-Adaptive Distance with Channel Quality (DADCQ) protocol [23], Link-based Distributed Multi-hop Broadcast (LDMB) [24] and Optimized Position-based Gossiping (OPbG) protocol [25] are some examples of this category.

\section{Comparison of routing based on network's topology and vehicle's position}

This section reviews in detail the evaluated routing protocols that includes routing based on network's topology and others based on vehicle's position.
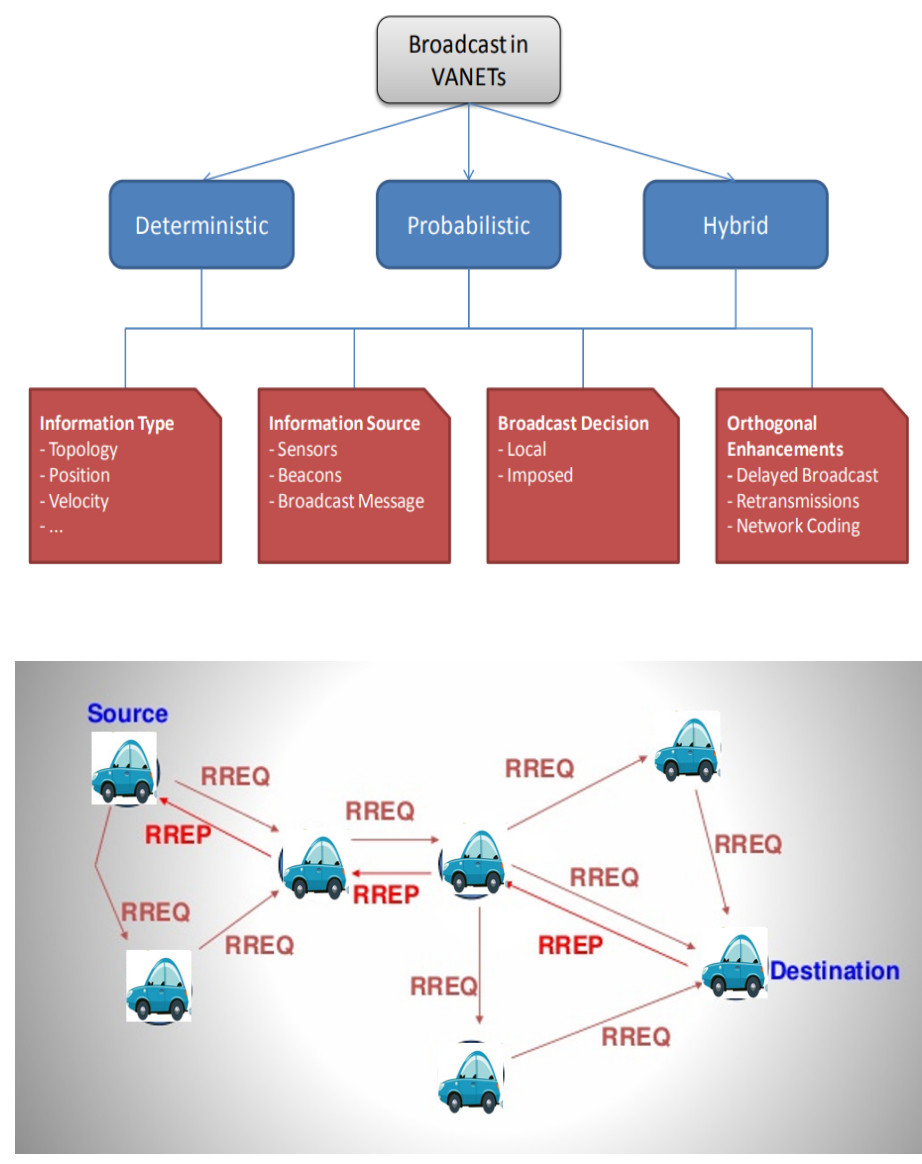

\subsection{Routing based on network's topology}

- AODV routing protocol. AODV [26] could be classified as unicast or multicast routing, the process adopted by this routing is that each paths are produced only on request. In fact, when a vehicle desires to transmit a packet by using the route discovery mechanism as Figure 3 shows, only the used routes are kept. In this case, the route discovery process causes significant delays before every data transmission to look for the path. AODV is also known by its capacity to diminish broadcasts, transmission latency and routing overhead. However, AODV suffers from the high end-to-end delay resulted by the route discovery process before every data transmission. Hence, the high E2ED is not suitable to vehicular networks in case of a crucial or dangerous information.

- OLSR routing protocol. In OLSR [27], each node constructs a global view of the network's topology by distributing after each specific time its routing table. In this routing technique, the concept of Multi point Relays (MPR) is utilized to relay control traffic. The MPR technique decreases the routing overhead issue by selecting only specific nodes to retransmit the control messages, which aids in optimizing the broadcast technique. 


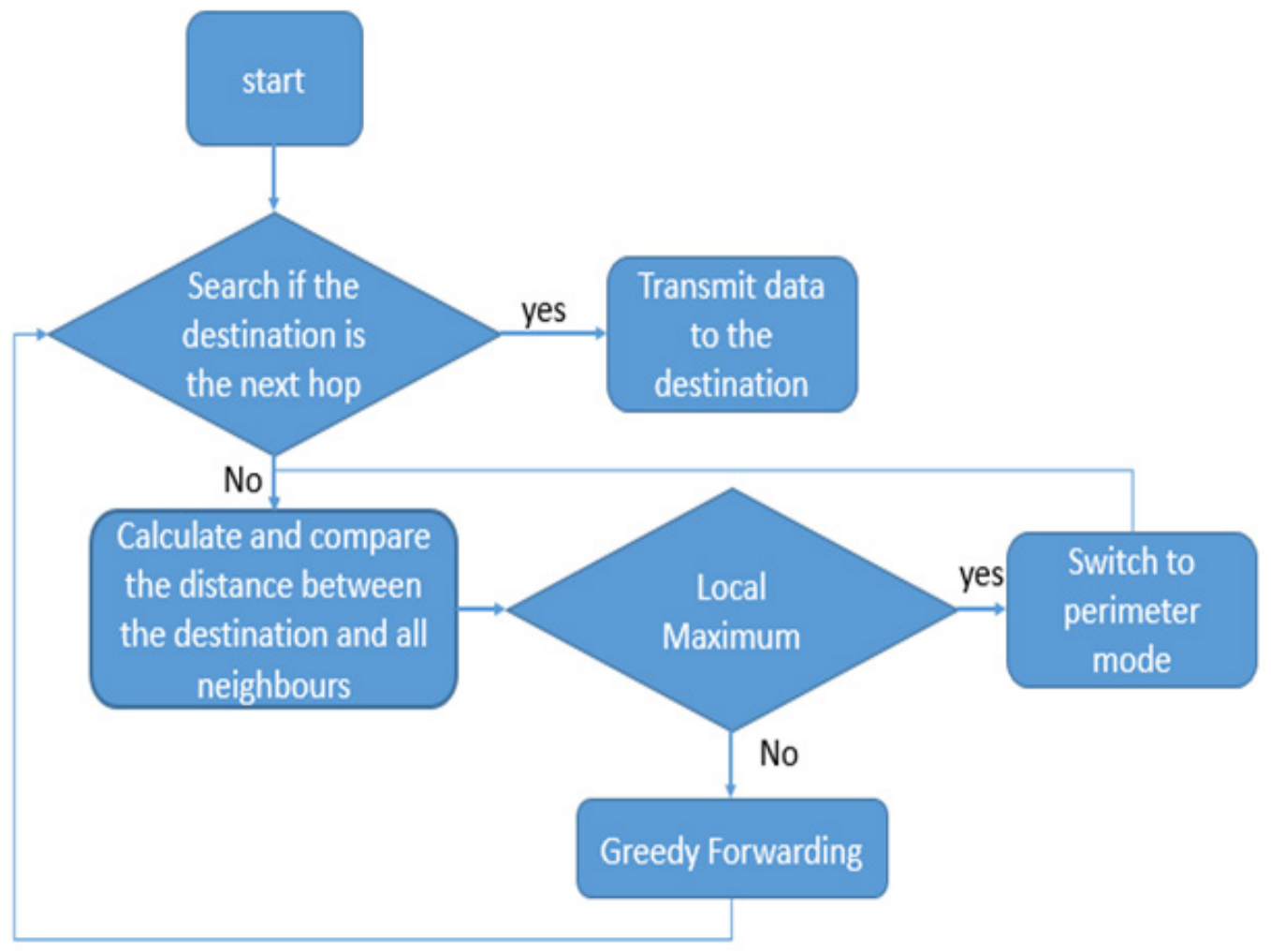

Figure 4 Flow diagram of route maintenance in GPSR

- DSDV routing protocol. In DSDV [28], [29], the update of routing tables of each nodes is done periodically to make available information about paths to each destination in the network at any time, even if the paths are at this time unused. In spite of the benefits of DSDV such as simplicity, loop free and no delay time caused by the discovery of the road technique. The DSDV still suffer from the high routing overhead caused by the periodic updates.

\subsection{Routing based on vehicle's position}

- GPSR [30] routing protocol. This routing protocol requires the availability of the physical location information of participating nodes using a location service like a GPS. In GPSR, the greedy forwarding (GF) technique is used by each relaying vehicle in order transmit data. In case of the local maximum problem, this technique fails; hence, the perimeter forwarding technique is applied to select another node through which a packet will travel. GPSR has many benefits: to transmit a data packet, a node necessities to memorize only the physical location of one hop neighbor. However, because of the features of high speed of vehicles, stale information of the neighbors' physical location are frequently contained in the sending nodes neighbor table. To be more clear, the strategy of GPSR is explained in Figure 4.

- GPCR routing protocol. Greedy Perimeter Coordinator Routing [31] protocol is an enhanced version of GPSR that uses a restricted greedy forwarding technique and a junction-based repair strategy in which routing decisions are made. Therefore, all forwarded packets must be sent to a vehicle located at an intersection or a junction; then this vehicle will transmit those packets to the closest neighbor of the recipient. GPCR has a great benefit that reduce the routing overhead problem and the end-to-end delay. Indeed, in the transmission process the election of the next hop is depends only on junction vehicles, which does not require any global or external information. Nevertheless, GPCR has some drawbacks that badly affect its performance. Actually, this technique assumes that there is at all times at least one vehicle at an intersection, which is not always the case.

\subsection{Simulation and comparison}

To simulate and evaluate the performance of the abovementioned routing protocols in VANETs, it would be very expensive to set up a network to test certain criteria. To remedy this problem, we will use network and traffic simulators. In this project, we realize a scenario where we test the performance of routing protocols by successively increasing the number of vehicles in the network, while the other network parameters are fixed.

Basically, our work was evaluated by computing the rate of three metrics as the received packet number, the end-to-end delay and the routing overhead. To ensure this task, a simulation was carried out under the network simulator NS3 [32] version 25 in combination with the road traffic simulator SUMO 0.25 [33] by using the operating system Ubuntu 14.04. We have also chosen the Open Street Map (OSM) [34] to download a real-world urban map for a part of Oujda city (Hay al Quds) in Morocco; after that, 

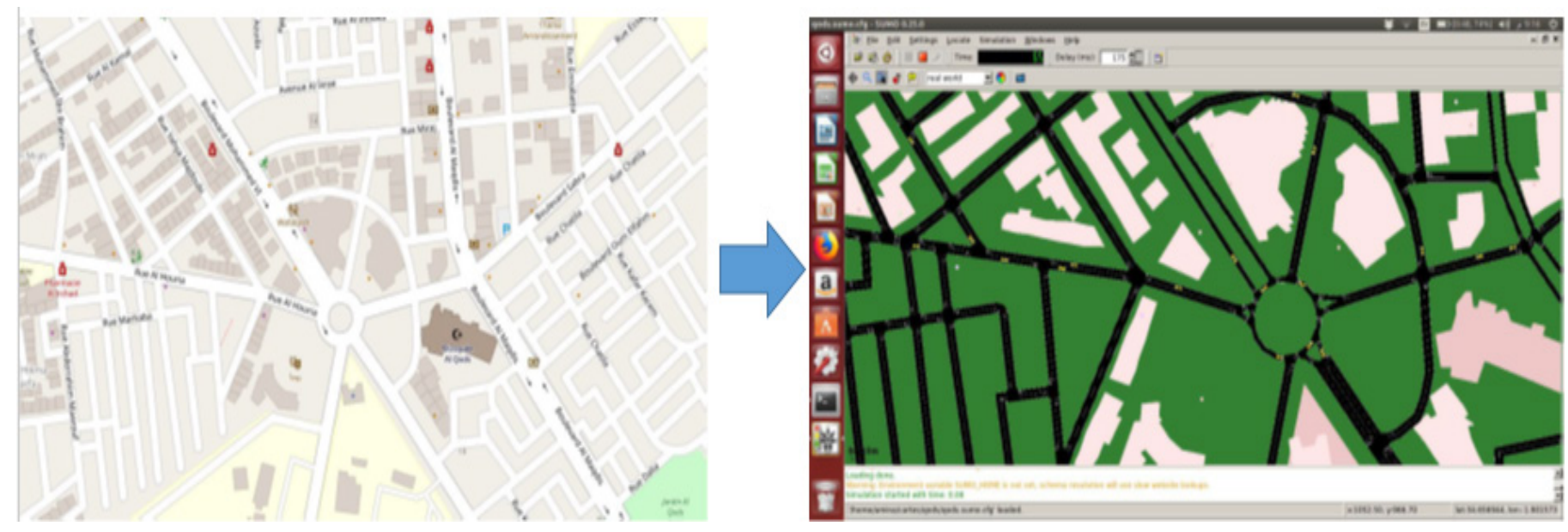

Figure 5 Hay Alquds Oujda map from OSM to SUMO

we have used SUMO to generate the traffic scenario as shown in Figure 5 .

In the used scenario, the number of nodes connected in the network varies. The simulation configuration is shown in Table 1 .

Table 1 Setting of experimental simulation parameters

\begin{tabular}{|l|l|}
\hline Parameters & Measures \\
\hline Routing Protocols & $\begin{array}{l}\text { AODV, OLSR, DSDV, GPSR, } \\
\text { GPCR }\end{array}$ \\
\hline Simulation Object & Hay-ElQuds Oujda city \\
\hline Number of nodes & $20,30,40,50,60,70,80,90$ \\
\hline Vehicles speed & Max $: 20 \mathrm{~m} / \mathrm{s}$ \\
\hline Simulation time & $100 \mathrm{~s}$ \\
\hline Simulation area & $1.7 * 1.5 \mathrm{~km}^{2}$ \\
\hline Packet size & 512 bytes \\
\hline Data type & Constant Bit Rate $(\mathrm{CBR})$ \\
\hline Transport protocol & UDP \\
\hline Propagation model & Two Ray Ground \\
\hline Mac protocol & IEEE $802.11 \mathrm{p}$ \\
\hline Channel & Channel/WirelessChannel \\
\hline Network interface & Phy/WirelessPhy \\
\hline Transmission range & $145 \mathrm{~m}$ \\
\hline Transmission power & $20 \mathrm{dBm}$ \\
\hline
\end{tabular}

- $\quad$ Packet Delivery Ratio (PDR)

According to the Figure 6, the results obtained confirm that DSDV and OLSR protocols have the best values of PDR. These values are increased when the vehicle's number increases, while they are reduced in case of AODV. Moreover, the curve stays stable For GPCR and GPSR protocols but with a moderate values of PDR. In fact, the huge number of radio obstacles in the proposed scenario are the main cause of this weak. Those obstacles increase the possibility of getting into the local maximum's trouble as explained in section IV. In addition, the PDR for GPSR and GPCR may be negatively affected because of the perimeter mode technique where the number of hops become high. For OLSR protocol, the good values obtained may be thanks to the use of Multi-Point Relay technique, which has a positive effect in case of dense and large networks.

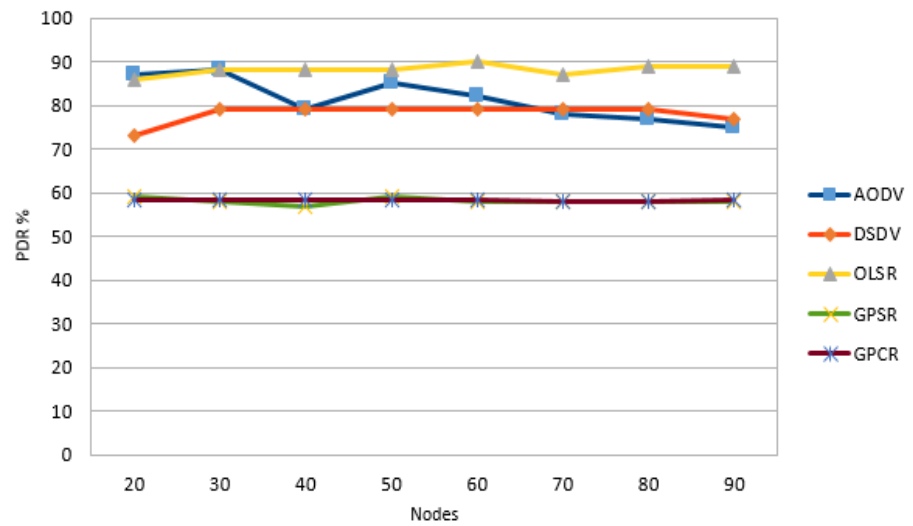

Figure 6 Packet Delivery Ratio Vs number of vehicles

- Average End-to-end Delay

Average End-to-end Delay or E2ED in short denotes the required time for transmitting a packet from the source node to the destination per the number of successfully transported and received packets. The protocol that has an average E2ED as low as possible is the good and the preferred one. To calculate the E2ED we used the equation (2).

$$
E 2 E D=\frac{\sum_{1}^{n} \text { receptionTime-SendTime }}{\text { ReceivedPackets }}
$$

From the graph showed in Figure 7, we approve that the OLSR protocol has the maximum values followed by DSDV. Conversely, GPSR and GPCR have the lowest values, which are almost the same for all the number of nodes. The achieved results for GPSR and GPCR could be thanks to the use of the greedy forwarding technique. By using this technique, the number of hops are reduced so as the necessary time for transmitting data packets until the endpoint vehicle. It is also clear, that AODV has the best values in case of the E2ED compared to DSDV and OLSR for low node density. However, for the high node density, the E2ED for AODV are high because of the use of the route discovery technique that introduces additional delay. 


\section{A. Bengag et al. / Advances in Science, Technology and Engineering Systems Journal Vol. 5, No. 1, 77-85 (2020)}

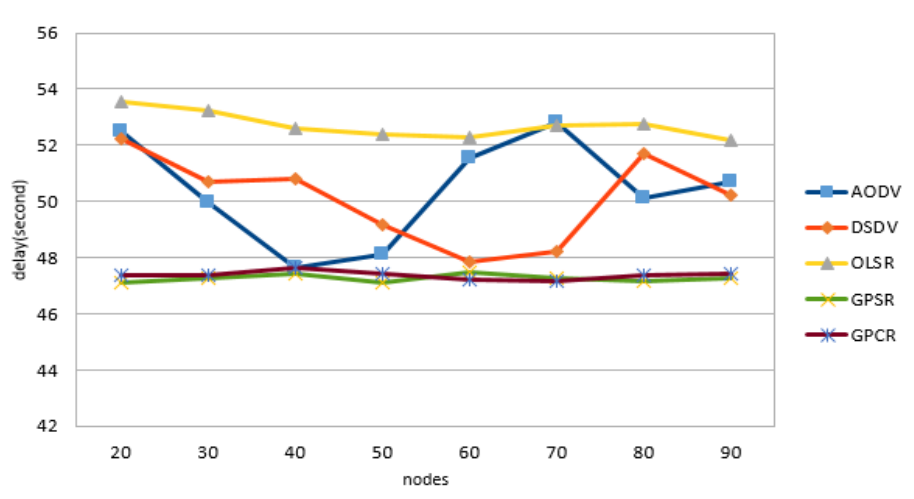

Figure 7 E2ED vs number of vehicles

- Throughput:

The graph in Figure 8 proves that OLSR has the best and highest values of throughput up to $18 \mathrm{kbps}$. In fact, the use of Multi point relay technique reduces the number of relaying nodes so as the routing overhead that helps in increasing the throughput. In case of DSDV and AODV, the throughput decreases as the density increases; this could be explained by the fact that DSDV broadcasts the entire routing table after fixed time interval, which introduces an additional overhead and affects its throughput. While GPSR and GPCR have the lowest throughput compared to the other three protocols.

- Routing Overhead:

The results obtained in Figure 9 show that the best results are in case of GPSR and GPCR protocols that have the smallest values of routing overhead. This result could be explained by the fact that GPSR and GPCR are based on the physical location of the destination that is contained in the routed packet so that the retransmitted node should not need to use the location service for a second time, which reduces the overhead. The highest control overhead is for AODV, as it broadcasts a large number of control message packets to maintain the route.

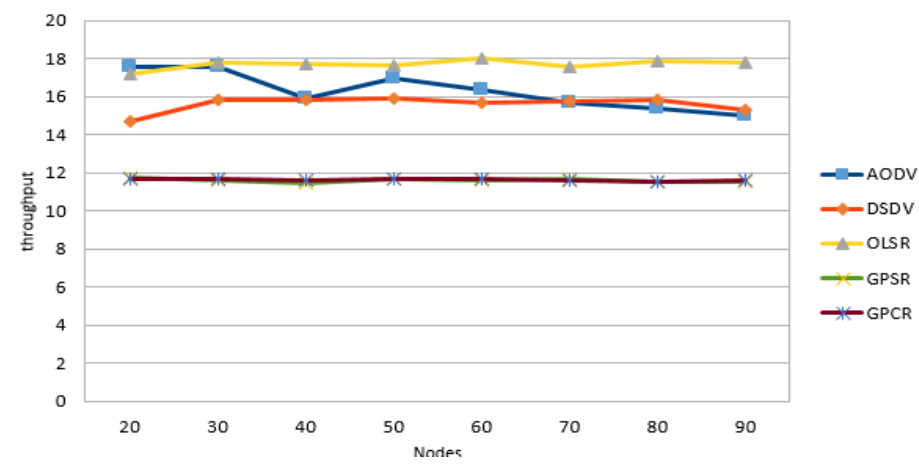

Figure 8 the throughput vs number of vehicles

\section{Work in progress}

In our proposed scenario, the performance evaluation above proves that OLSR has the best results in case of PDR and throughput. At the same time, GPSR and GPCR have the best results in case of overhead and E2ED. Indeed, the last two protocols are based on the destination's position that is carried in the packet, so the transferring vehicles do not need to use the location service for a second time and thus reduce the issue of routing overhead.

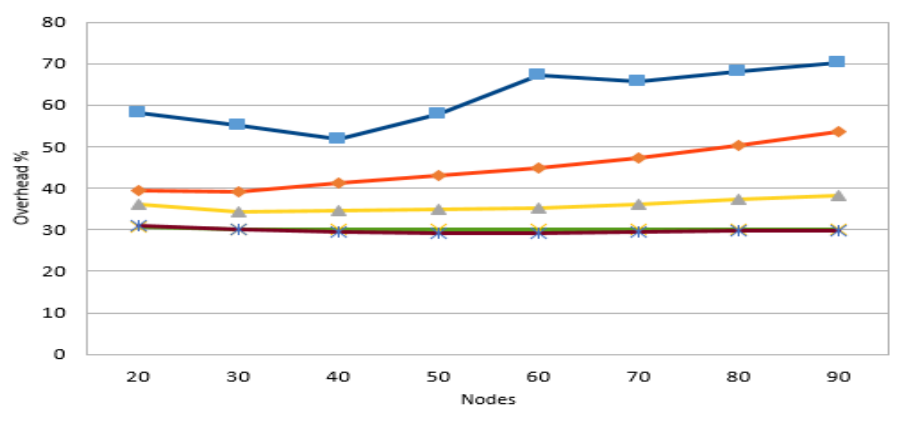

Figure 9 routing overhead vs number of vehicles

GPSR and GPCR have a moderate amount of PDR and Throughput. Hence, to optimize the ratio of the mentioned parameters, we proposed a new algorithm that improves the classical technique of greedy forwarding. This novel technique is used to select the best relaying vehicle candidate by taking into consideration four main metrics: the angle direction, the speed variation, the density of each nodes in addition to the distance between the next-hop candidate vehicle and the destination vehicle.

Our main contribution consists in suggesting a new greedy forwarding strategy based on a simple weighted function (WF), which uses the four last mentioned metrics. Then, an improved GPSR and GPCR protocols will be provided based on our proposed strategy. More specifically, the chosen of the best relaying node candidate is done by selecting the neighbor node whose header angle is closest to the destination node. Therefore, the connection between the source and the destination consists of a series of nodes that move approximately in the same direction. The main goal of this concept is to maintain the connection between the nodes as long as possible, in order to reduce the problem of link breakage. This is in contrast to the simple greedy forwarding, which only considers the distance between the next hop and the destination and does not take into account the connection lifetime. The new technique also could enhance the stability of the routing link, which mitigates the influence of high dynamic topology in VANETs networks.

In GPSR and GPCR, without considering moving direction of vehicles while looking for the next optimal hop, the routing techniques could produce an erroneous decision to transmit a data packet, which could result in a high packet loss issue. In addition, the regularly link breakage problem may also disturbs the quantity of delivered packets, particularly in highway environment where the speed is very high. Basically, when a node receives a Hello packet to up-to-date its neighbor's list, it computes the best next hop and then forwards packet. Thus, during this process, the selected neighbor could be outside the transmission range and the packet cannot reach it due to the high network's mobility. In order to solve those issues that lead to performances degradation, we 


\section{A. Bengag et al. / Advances in Science, Technology and Engineering Systems Journal Vol. 5, No. 1, 77-85 (2020)}

thought to use other parameters as mentioned above like the speed, the density of neighbors and the distance between all participating nodes and the destination during routing operation in addition to the moving direction. Furthermore, a simple weighted function (WF) will be applied on each relaying candidate vehicle, then the relaying candidate node that has the lowest value of the WF will be selected.

The proposed technique resolves mainly three problems in vehicular networks, which could be proved through theoretical analysis:

1. The problem of void area is resolved by taking into account the density (number of neighbors) of the selecting node. In Figure 10, we present the density parameter or the number of neighbors of a selected node. Basically, vehicle A has the vehicles B to $\mathrm{F}$ as neighbors because they belong to its transmission range.

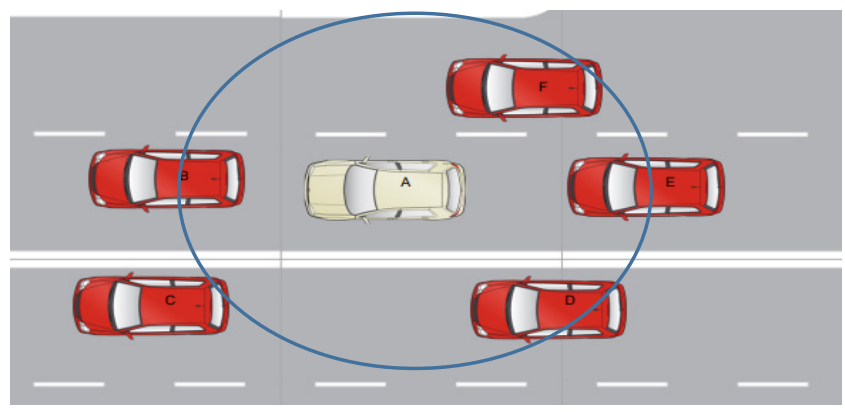

Figure 10 neighbors of the node A (density)

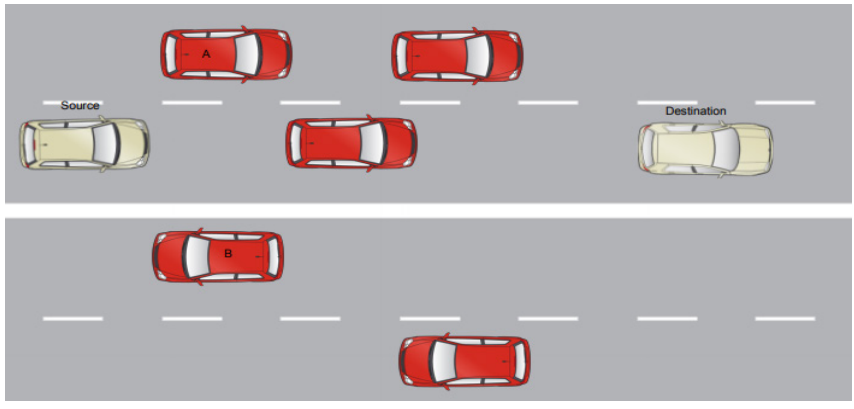

Figure 11 void area problem

Indeed, by using the proposed technique the node that has the big number of neighbors increases the probability to be chosen as the next hop. Figure 11 presents an example in which the source will choose $A$ as the next hop because it has three neighbors while $\mathrm{B}$ has no neighbors.

2. The problem of direction of nodes in VANETs will be fixed by taking into account the angle direction calculated between the next hop and the destination node. As the Figure 12 shows, the source node will choose A as the next hop by applying our proposed technique because it has almost the same direction as the destination. The angle direction between the next hop and the destination is calculated by using our proposed formula (3).

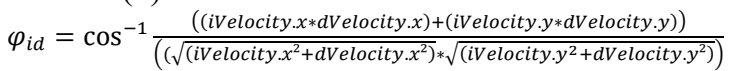

Where iVelocity is the velocity of the next hop candidate and dVelocity is the destination velocity. The rational between the concepts of the angle direction is to maintain the connection between vehicles as long as possible by choosing the small value of all calculated $\varphi_{-}$id.

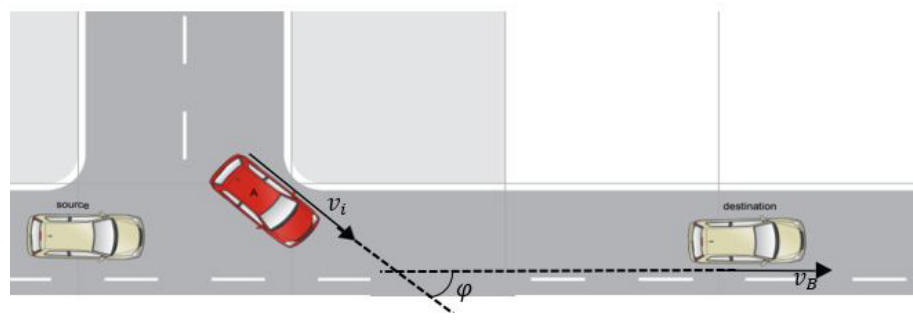

Figure 12 the angle direction $\varphi$

\section{Conclusion}

In this paper, we have provided a taxonomy of routing protocols in VANETS. Then we have given a quantitative analysis of the most known routing protocols for vehicular networks, using two simulation tools SUMO as a road traffic simulator and NS-3 as a network simulator, to determine the benefits and challenges of each algorithm.

From the results of the simulation, we have picked up the feebleness of GPSR and GPCR in terms of PDR and throughput. Based on the given results and to enhance the performance of those protocols, we have proposed a technique that combines the simple greedy forwarding technique and some other parameters selected carefully such as the direction, the speed and the density to choose the next relaying vehicle.

As a future work, we plan to develop our proposed technique to enhance GPSR and GPCR then prove their performance compared to the original GPSR and GPCR.

\section{Conflict of Interest}

The authors declare no conflict of interest.

\section{Acknowledgment}

This research is performed inside the MATSI Lab., ESTO, University Mohammed First, Oujda (Morocco). 


\section{References}

[1] B. Amina and E. Mohamed, "Performance Evaluation of VANETs Routing Protocols Using SUMO and NS3," in Colloquium in Information Science and Technology, CIST, 2018, vol. 2018-Octob, pp. 525-530.

[2] A. Bengag and M. El Boukhari, "Classification and comparison of routing protocols in VANETs," in 2018 International Conference on Intelligent Systems and Computer Vision, ISCV 2018, 2018, vol. 2018-May.

[3] E. Spaho, M. Ikeda, L. Barolli, and F. Xhafa, "Performance comparison of OLSR and AODV protocols in a VANET crossroad scenario," Lect. Notes Electr. Eng., vol. 253 LNEE, pp. 37-45, 2013.

[4] S. Singh, P. Kumari, and S. Agrawal, "Comparative Analysis of Various Routing Protocols in VANET," 2015 Fifth Int. Conf. Adv. Comput. Commun. Technol., pp. 315-319, 2015.

[5] B. V. Coutinho, E. C. G. Wille, and H. I. Del Monego, "Performance of routing protocols for VANETs," Proc. 9th Int. Conf. Ubiquitous Inf. Manag. Commun. - IMCOM '15, pp. 1-6, 2015.

[6] R. Bala and C. R. Krishna, "Scenario Based Performance Analysis of AODV and GPSR Routing Protocols in a VANET," in 2015 IEEE International Conference on Computational Intelligence \& Communication Technology, 2015, pp. 432-437.

[7] A. Ashtaiwi and S. Abdusadik, "Performance Comparison of Position based Routing Protocols for VANETs," pp. 78-84, 2017.

[8] G. Sallam and A. Mahmoud, "Performance Evaluation of OLSR and AODV in VANET Cloud Computing Using Fading Model with SUMO and NS3," 2015 Int. Conf. Cloud Comput. ICCC 2015, pp. 1-5, 2015.

[9] Z. J. Haas, M. R. Pearlman, and P. Samar, "The Zone Routing Protocol (ZRP) for Ad Hoc Networks," draftietfmanetzonezrp02 txt. p. 11, 2002.

[10] J. Liu, J. Wan, Q. Wang, P. Deng, K. Zhou, and Y. Qiao, "A survey on position-based routing for vehicular ad hoc networks," Telecommun. Syst., vol. 62 , no. 1, pp. 15-30, 2016.

[11] Jing Zhao and Guohong Cao, "VADD: Vehicle-Assisted Data Delivery in Vehicular <emphasis emphasistype="italic" $>$ Ad Hoc $</$ emphasis $>$ Networks," IEEE Trans. Veh. Technol., vol. 57, no. 3, pp. 1910-1922, May 2008.

[12] P.-C. Cheng, K. C. Lee, M. Gerla, and J. Härri, "GeoDTN+Nav: Geographic DTN Routing with Navigator Prediction for Urban Vehicular Environments," Mob. Networks Appl., vol. 15, no. 1, pp. 61-82, Feb. 2010.

[13] Y. Lin, Y. Chen, and S. Lee, "Routing Protocols in Vehicular Ad Hoc Networks A Survey and Future Perspectives.," J. Inf. Sci. ..., vol. 932, no. c, pp. 913-932, 2010.

[14] B. Marzak, H. Toumi, E. Benlahmar, and M. Talea, "Analysing Clustering Routing Protocols Performance for Vehicular Networks," Proc. 2nd Int. Conf. Big Data, Cloud Appl. - BDCA'17, pp. 1-7, 2017.

[15] Y. L. Y. Luo, W. Z. W. Zhang, and Y. H. Y. Hu, "A New Cluster Based Routing Protocol for VANET," Networks Secur. Wirel. Commun. Trust. Comput. NSWCTC 2010 Second Int. Conf., vol. 1, pp. 176-180, 2010.

[16] S. Allal, S. Allal, and S. Boudjit, "Geocast Routing Protocols for VANETs : Survey and Geometry-Driven Scheme Proposal Geocast Routing Protocols for VANETs: Survey and Geometry-Driven Scheme Proposal," no. February 2013, 2015.

[17] A. Bachir and A. Benslimane, "A multicast protocol in ad hoc networks inter-vehicle geocast," IEEE Semiannu. Veh. Technol. Conf., vol. 4, pp. 2456-2460, 2003.

[18] Y. S. Chen and Y. W. Lin, "A mobicast routing protocol with carry-andforward in vehicular ad hoc networks," Int. J. Commun. Syst., vol. 27, no. 10, pp. 1416-1440, 2014.

[19] S. Zeadally, R. Hunt, Y.-S. Chen, A. Irwin, and A. Hassan, "Vehicular ad hoc networks (VANETS): status, results, and challenges," Telecommun. Syst., vol. 50, no. 4, pp. 217-241, 2012.

[20] B. Williams and T. Camp, "Comparision of broadcasting techniques," pp. 194-205, 2002.

[21] B. Bako, "Efficient Information Dissemination in VANETs," 2016.

[22] O. Tonguz, N. Wisitpongphan, F. Bai, P. Mudalige, and V. Sadekar, "Broadcasting in VANET," in 2007 Mobile Networking for Vehicular Environments, MOVE, 2007, pp. 7-12.

[23] M. Slavik and I. Mahgoub, "Spatial distribution and channel quality adaptive protocol for multihop wireless broadcast routing in VANET," IEEE Trans. Mob. Comput., 2013.

[24] Q. Yang and L. Shen, "A Multi-Hop Broadcast scheme for propagation of emergency messages in VANET," in International Conference on Communication Technology Proceedings, ICCT, 2010.

[25] B. Bako, E. Schoch, F. Kargl, and M. Weber, "Optimized position based gossiping in VANETs," in IEEE Vehicular Technology Conference, 2008.
[26] C. E. Perkins and E. M. Royer, "Ad-hoc on-demand distance vector routing," in Proceedings - WMCSA'99: 2nd IEEE Workshop on Mobile Computing Systems and Applications, 1999, pp. 90-100.

[27] T. Clausen and P. Jacquet, "Optimized Link State Routing Protocol (OLSR)," Ietf $R f c 3626$, p. 75, 2003.

[28] C. E. Perkins and P. Bhagwat, "Highly dynamic Destination-Sequenced Distance-Vector routing (DSDV) for mobile computers," ACM SIGCOMM Comput. Commun. Rev., vol. 24, no. 4, pp. 234-244, 1994.

[29] G. He, "Destination-sequenced distance vector (DSDV) protocol," Netw. Lab. Helsinki Univ. Technol., pp. 1-9, 2002.

[30] B. Karp and H. Kung, "GPSR: Greedy Perimeter Stateless Routing for wireless networks," ACM MobiCom, no. MobiCom, pp. 243-254, 2000.

[31] C. Lochert, M. Mauve, H. Füßler, and H. Hartenstein, "Geographic routing in city scenarios," ACM SIGMOBILE Mob. Comput. Commun. Rev., vol. 9, no. 1 , p. 69, 2005.

[32] R. Manual et al., "ns-3 Tutorial," System, no. January, pp. 1-46, 2011.

[33] M. Behrisch, L. Bieker, J. Erdmann, and D. Krajzewicz, "SUMO Simulation of Urban MObility," Iaria, no. c, pp. 55-60, 2011.

[34] OpenStreetMap Foundation, "OpenStreetMap," Open Database License (ODbL). 2013. 